\title{
AC Loss Measurements with a Cryocooled Sample
}

\author{
S.W. Schwenterly, J.A. Demko, J.W. Lue, M.S. Walker, C.T. Reis, D.W. Hazelton, Xin Shi and M.T. Gardner
}

\begin{abstract}
A new cryostat cooled by a closed-cycle Cryomech GB-37 cryocooler for superconductor measurements at temperatures down to $20 \mathrm{~K}$ is described. The sample is conductively coupled to the cold stage so as to minimize vibration and thermal stresses. AC losses have been measured calorimetrically in several HTSC coils that have been wound to simulate sub-scale transformer winding pairs. Stable temperatures down to $20 \mathrm{~K}$ were reached on these coils, allowing measurements at practical levels of ac current and $I_{c}$. By using short ac current pulses, losses on individual turns could be resolved. Results are reported mainly to showcase the apparatus, measurement procedure and analytical approach.
\end{abstract}

Index Terms - Cryogenics, High-Temperature Superconductors, Loss Measurement, Transformer Windings.

\section{INTRODUCTION}

$\mathrm{O}$ NE of the major components of the thermal load in superconducting ac utility power equipment, such as transformers, is the ac loss generated in the superconducting windings. Since this loss occurs at low temperature, its effective value is increased by a factor of 20 to 50 due to the room-temperature refrigeration power required to remove it [1]. The ac loss also increases rapidly when the equipment is operated in an overload condition.

This paper describes the apparatus and reports results of ac loss measurements in support of a U.S. Department of Energy Superconductivity Partnership Initiative (SPI) to develop high-temperature superconducting utility power transformers. The SPI team is led by Waukesha Electric Systems (WES) with team partners IGC-SuperPower (IGC), Rochester Gas and Electric Company, and Oak Ridge National Laboratory. In Phase I of this SPI, a 1-MVA hightemperature superconducting (HTS) transformer was designed, built, and tested [2]. The HTS windings were designed to operate near $25 \mathrm{~K}$. In tests on this transformer, the cryogenic systems worked well. However, ac losses become an increasingly important issue as more conductor turns are used and greater winding fields are experienced in transformers of higher power rating. The SPI is now in Phase II, with the objective of building a 5/10-MVA HTS transformer and testing it on the utility power grid to power

Manuscript received September 18, 2000. This work was supported in part by the U.S. Department of Energy Superconductivity Partnership Initiative, Contract \#DE-FC36-98GO10282, and sponsored by U. S. Department of Energy, Energy Efficiency and Renewable Energy, Superconductivity Program for Electric Power, under contract No. DEAC05-00OR22725 with UT-Battelle, LLC.

S.W. Schwenterly, J.A. Demko, and J.W. Lue are with Oak Ridge National Laboratory, Oak Ridge, TN 37831 USA (telephone: 865-5741460, e-mail: schwenterlsw@ornl.gov).

M.S. Walker, C.T. Reis, D.W. Hazelton, Xin Shi, and M.T. Gardner are with IGC-SuperPower, Schenectady, NY 12304 USA (telephone: 518-3461414, e-mail: mwalker@IGC.com). the WES main transformer manufacturing plant [3]. This transformer will also operate with windings at temperatures well below $77 \mathrm{~K}$.

The tests described here were conducted as part of a program to develop lower loss coils for this transformer and to predict the ac losses that may be expected for commercial HTSC superconducting transformer designs. Test coils were provided by IGC-SuperPower. While the detailed nature of these coils and the magnitudes and dependencies of their losses are confidential and proprietary, the apparatus and techniques used to obtain the loss measurements are new and potentially broadly effective for many applications, and are believed to be of considerable interest to the technical community. Test results are therefore reported using arbitrary units in a way that is mainly intended to showcase the apparatus and experimental and analytical approach. The apparatus is particularly useful in its ability to measure losses in individual coil turns and to easily provide temperatures below $77 \mathrm{~K}$, which elevates the $\mathrm{I}_{\mathrm{c}}$ 's and allows the operating currents of today's conductors to reach the practical levels expected for conductors of the future.

The new cryostat incorporates a Cryomech GB-37 cryocooler which allows tests on superconducting coils and short samples down to temperatures as low as $10 \mathrm{~K}$. A calorimetric method similar to that originally proposed by Schmidt and Specht [4] was used to measure the ac losses in test coils in the $77 \mathrm{~K}$ to $20 \mathrm{~K}$ temperature range. The coils were mounted in high vacuum and thermally coupled to the cryocooler. Temperature sensor voltages on the various turns were monitored while roughly 10 -sec pulses of ac current were applied. The initial $d V / d t$ slopes for each current pulse were converted to ac loss power by comparing them with the slopes observed with known heat input from a heater cowound with the conductor. This use of initial slopes is in contrast to the use of equilibrium temperature rise by Schmidt and Specht and most other workers. It allows fast and accurate spatial resolution of local losses on individual conductor turns before thermal diffusion alters the initial temperature gradients. If the heater and conductor are in close thermal contact, definitive loss measurements can be made with no information required about either the effective heat capacity of the test coil or its thermal contact to the refrigerator. Experimental details are given in Section IV.

\section{APPARATUS}

Figure 1 shows a diagram of the cryostat. The test coil and cryocooler are surrounded by high vacuum. Considerable care was taken to allow for differential thermal contraction between the cryocooler cold head and the test coil suspension. The test coil is supported from below by a copper disc that hangs from the cryostat top flange on three G-10 fiberglass rods. A similar copper disc above the test 
2000 Applied Superconductivity Conference, Paper \# 3LF02

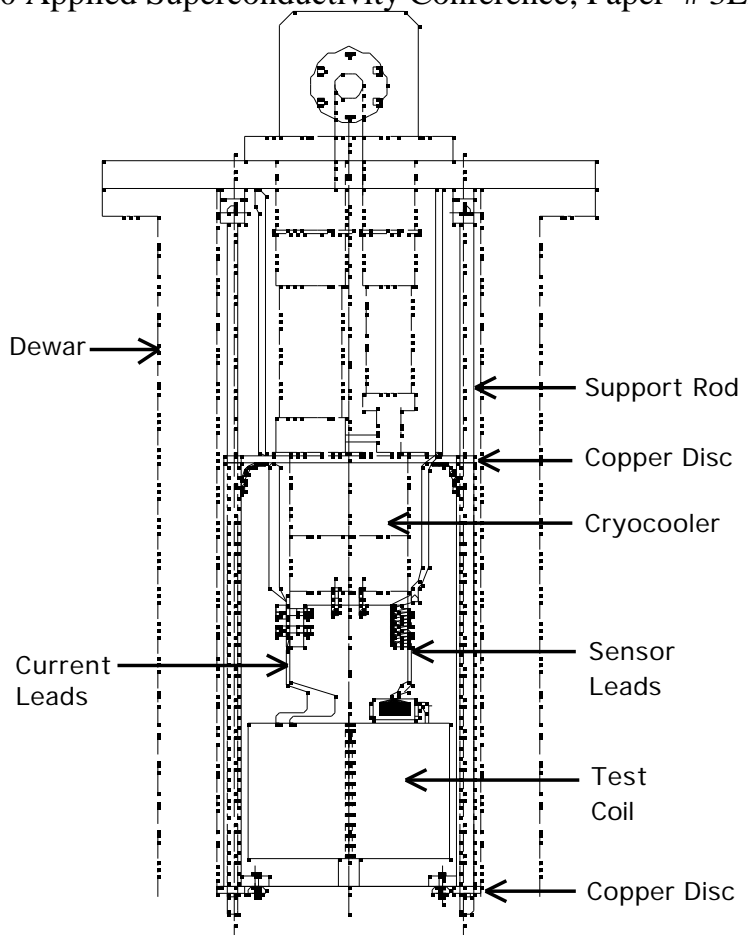

Fig. 1. Diagram of cryostat.

coil is bolted to the first stage of the cryocooler. Three copper tubes slide on the fiberglass rods and form a thermal link between the two copper discs. These tubes are joined to the upper copper disc by flexible copper straps and bolted to the lower disc. Thus, the whole assembly of copper discs and tubes is maintained at roughly $40 \mathrm{~K}$ by the cryocooler first stage. A G-10 spindle isolates the test coil from the lower copper disc.

The copper foil current leads on the test coil are insulated with Kapton tape and clamped to the cryocooler second stage. They are the main thermal link to the coil conductors and mandrels. Both the current leads and the test coil instrumentation leads are also thermally grounded to the cryocooler first stage. For additional thermal linkage to the test coil structure, four $10-\mathrm{cm}$-wide straps of 0.025 -cm-thick copper foil are clamped to the cryocooler second stage and bound tightly to the outer cylindrical surface of the test coil. Grease and/or indium foil is used in all mechanical joints to improve thermal contact. Silicon diode temperature sensors are mounted on both cryocooler stages. Fig. 2 is a photo of the cryostat with the sample installed.

The cryostat is mounted in a standard laboratory liquid helium dewar, which is used as a vacuum vessel. The liquid nitrogen shield in the dewar vacuum jacket provides additional heat shielding.

\section{TEST COILS}

Three test coils have been provided by IGC. Fig. 3 is a photo of a typical test coil. Each coil has concentric primary and secondary windings in a configuration similar to that in the 5/10-MVA transformer. Since there is no iron core, the two windings are connected in series to ensure that they have equal currents. The two windings are connected to buck each other's magnetic flux, so that there is net flux primarily only in the gap. This arrangement realistically reproduces the flux

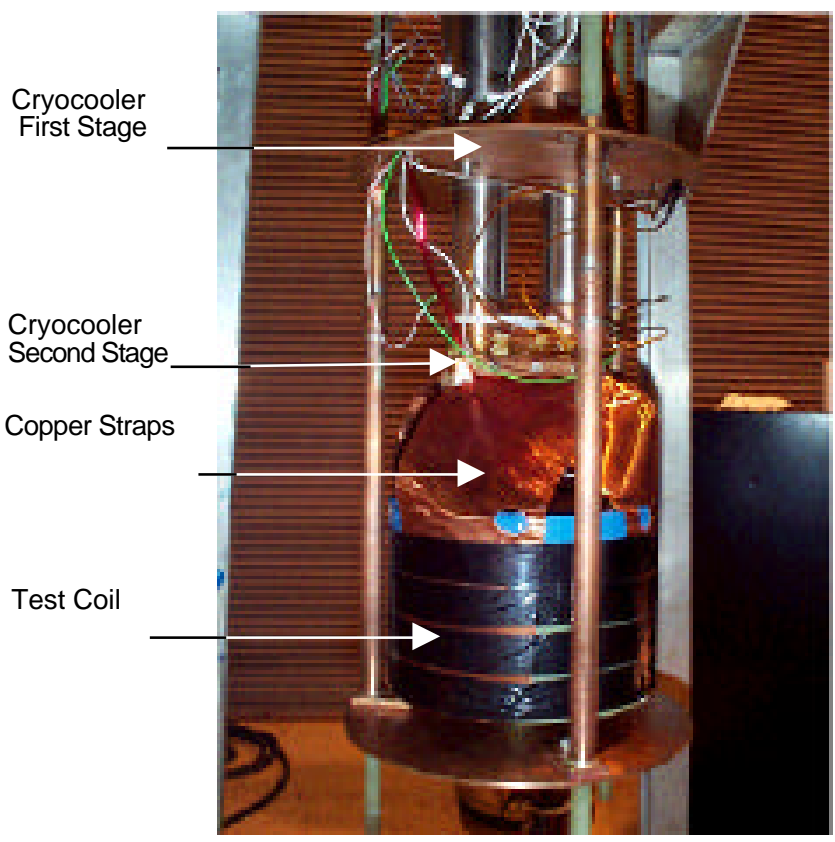

Fig. 2. Photo of cryostat.

configuration in the windings of a transformer. The windings are supported by G-10 fiberglass mandrels, and enclosed in a cylindrical G-10 shroud with an overall diameter of $18.5 \mathrm{~cm}$ and a height of $12.5 \mathrm{~cm}$. The test coils have wide copper foil leads that can be easily clamped to the cryocooler cold stage to allow direct cooling of the conductor.

The coils are instrumented with voltage taps across various sections of the windings and with eight Allen-Bradley carbon resistors mounted on individual turns as thermometers. A calibrated Scientific Instruments RuO thermometer was also installed to provide an absolute temperature check for the carbon resistors. The bulk of the instrumentation is on the outer winding in each test coil. The outer winding also has a heater wound on top of the superconductor to supply a known heat input for calibration of the ac loss measurements. This heater is designed not to produce any appreciable eddy

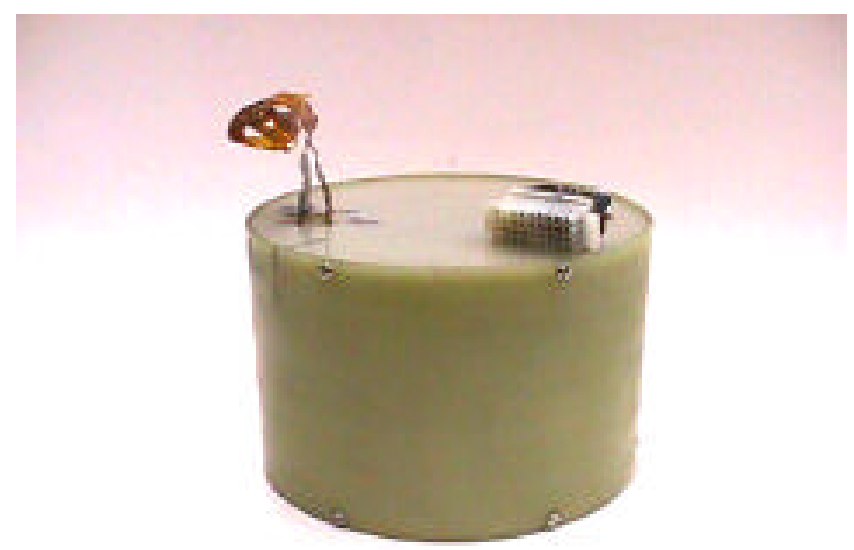

Fig. 3. Photo of Test Coil. 
2000 Applied Superconductivity Conference, Paper \# 3LF02 current heating during the ac current tests.

Test Coil 1 is wound with surface coated $\mathrm{Bi}-\mathrm{Sr}-\mathrm{Ca}-\mathrm{Cu}-\mathrm{O}$ (BSCCO)-2212 tape conductor with a self-field critical current of 8-10 A at $77 \mathrm{~K}$. This conductor is of interest as a close simulation of the behavior expected for future secondgeneration surface coated Y-Ba-Cu-O (YBCO)-123 tapes. Test Coils 2 and 3 are wound with multifilamentary BSCCO-2223 tape with a self-field critical current of about $24 \mathrm{~A}$ at $77 \mathrm{~K}$. This tape is narrower than the BSCCO-2212 tape, allowing a more compact winding with higher current density.

Theoretical studies by IGC have indicated that the coil configuration has a strong effect on the its ac losses. Two different proprietary winding configurations have been identified to be particularly promising for producing low losses. These configurations are designated as 1 and 2 . Configuration 1 is used in Test Coils 1 and 3 , and Configuration 2 is used for Test Coil 2. Table 1 summarizes the parameters of the test coils.

\section{PROCEDURE}

The cryostat with the mounted test coil was installed in the dewar and pumped down to a rough vacuum of about 50 microns. As the cryocooler cooled down, the pressure soon fell below 1 micron. Since the test coil was cooled only by its leads and through the G-10 structure, cooldown was slow and about $10 \mathrm{hr}$ was needed to reach the minimum temperature of 20-25 K. This did not cause problems because the cryocooler could be operated overnight unattended. A heater on the cryocooler cold stage was used to reach higher temperatures. .If the liquid nitrogen in the dewar shield was allowed to boil away, the test coil warmed up without the heater to 40-45 K. It was also found that the test coil could be heated by applying ac current to it and using the ac loss as a heating mechanism. The temperature distribution in the test coils was uniform within $\pm 2 \mathrm{~K}$ at the start of each ac current run. Overall operation of the experiment was much easier and less labor-intensive than earlier ac loss tests with a liquidhelium-cooled variable-temperature cryostat. This along with the excellent temperature stability and unattended operation capability of the cryocooler made its initial cost well worthwhile.

Data were taken at temperatures near $20 \mathrm{~K}, 30 \mathrm{~K}$, and $40 \mathrm{~K}$. The data acquisition system was set to scan every $3 \mathrm{sec}$. When the desired temperature was established, $60-\mathrm{Hz}$ ac current pulses of duration 10-12 sec were applied. Pulses were applied every $100 \mathrm{sec}$ to allow the sample to re-cool. Calibration runs using known heat inputs from the heater were performed at each temperature. The heater pulses were also limited to 10 or $12 \mathrm{sec}$ to simulate the ac heating as closely as possible and to limit the total heating on the test coil. To increase the speed of the data system, the resistor voltages were logged directly without conversion to

TABLE I

TEST COILS

\begin{tabular}{ccc}
\hline \hline Test coil & Conductor / width & $\begin{array}{c}\text { Winding } \\
\text { Configuration }\end{array}$ \\
\hline 1 & BSCCO-2212/6 mm & 1 \\
2 & BSCCO-2223/4 mm & 2 \\
3 & BSCCO-2223 / 4 mm & 1 \\
\hline \hline
\end{tabular}

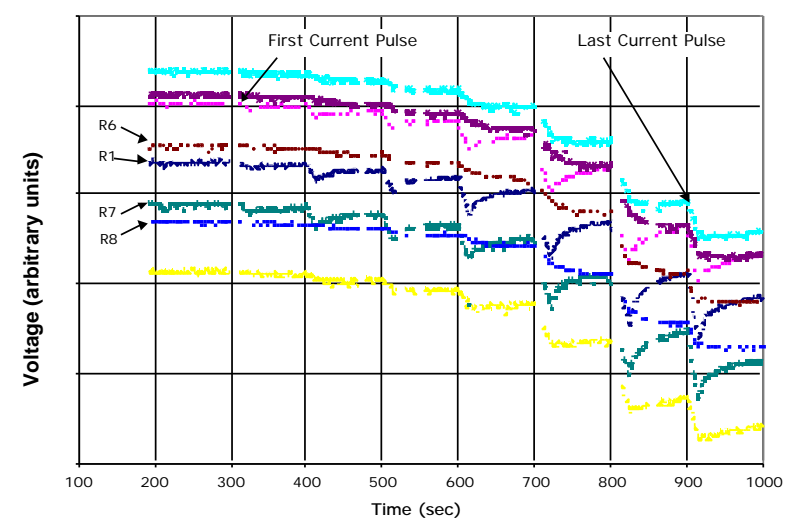

Fig. 4. Resistance thermometer voltages vs. time for Test Coil 1 at $30 \mathrm{~K}$.

temperatures during a test run. The initial slopes of the voltage vs time curves were determined by performing a least-squares fit to the logged data during each current or heater pulse. The slopes due to any drift in temperature just prior to a heater pulse or ac current pulse were subtracted from those observed during the pulses.

\section{RESULTS}

As described in the Introduction, these test results are presented primarily to illustrate this new and effective apparatus and measurement technique without revealing the details of the coil designs and the magnitude and functional dependencies of the losses, which are proprietary. Results are therefore presented in arbitrary units. Within these limitations, the measurement and analytical techniques and the physical responses of the coils to ac loss pulses and calibration pulses are described as completely as possible.

Figure 4 shows a typical plot of the resistance thermometer voltages vs time for short duration pulses of ac current at $30 \mathrm{~K}$ with Test Coil 1. Each time the ac current was applied, sharp drops in resistance were seen, corresponding to heating of the resistor by the ac losses in the adjacent conductor. The strongest heating was seen on resistors R1 and R7, which were on the top and bottom turns of the outer coil and in considerable perpendicular magnetic field. The heating was noticeably less severe on R6 and R8. These two resistors were mounted on the middle turn of the outer and inner winding. These turns had the lowest perpendicular field and thus the lowest ac losses. The test coil temperature increased by about $3 \mathrm{~K}$ during the run. It should be noted that this temperature increase is not characteristic of that expected for the actual transformer windings, which will be thermally coupled to the cryocooler much more tightly than the test coil.

Figure 5 shows a similar plot of the resistor voltages during a calibration run with the heater on Test Coil 1. A clear slope can be seen with less than $0.1 \mathrm{~W}$ on the heater. In contrast to the situation with ac current, the heater warmed all the turns rather uniformly, as expected. The heater power was divided by the heater length to produce a relationship between the power per unit length and the voltage change rate $d V / d t$ for each resistor on its individual turn. As expected, this relationship was approximately linear. The resulting conversion factor between heater power per unit length and 
2000 Applied Superconductivity Conference, Paper \# 3LF02

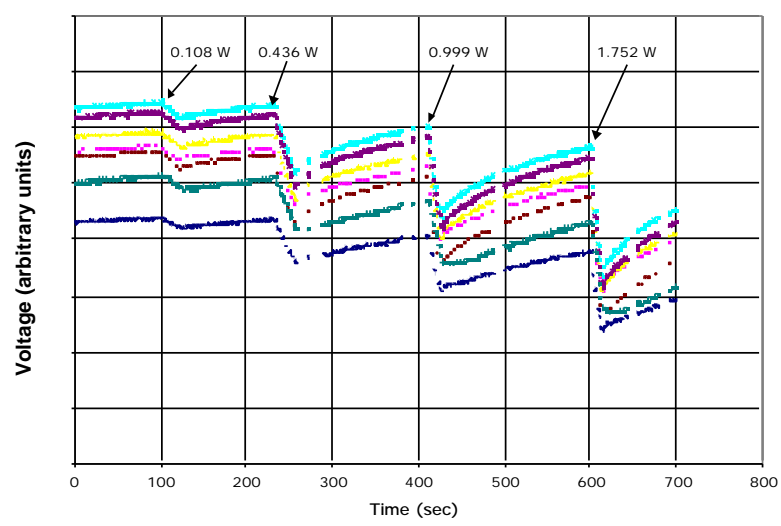

Fig. 5. Resistor voltages during a calibration run for Test Coil 1 at $30 \mathrm{~K}$.

$d V / d t$ was multiplied by the $d V / d t$ observed on each resistor during the ac current tests, to obtain the ac loss rate per unit length on the turn where the resistor is located. Loss sensitivities of less than $10 \mathrm{~mW} / \mathrm{m}$ were obtained.

Figure 6 shows the comparative performance of the three test coils at one temperature and current. The plots show the losses per unit length in arbitrary (but consistent) units vs. the turn location in the winding in percent. The zero percent points were at the end turns on the outer winding, and had the highest losses. The losses decreased on turns successively closer to the center of the winding. These low center losses are characteristic of the losses designed and expected for nearly the entire primary and secondary coils in the 5/10MVA transformer. Test Coil 2 showed a peak in the losses $25 \%$ below the top end of the winding. This anomalous behavior was traced to variations in the conductor critical current. The dc V-I curves for this sample showed that the conductor near the loss peak had considerably lower than average critical current compared to the rest of the sample, and the conductor near turn 1 had considerably higher than average critical current.

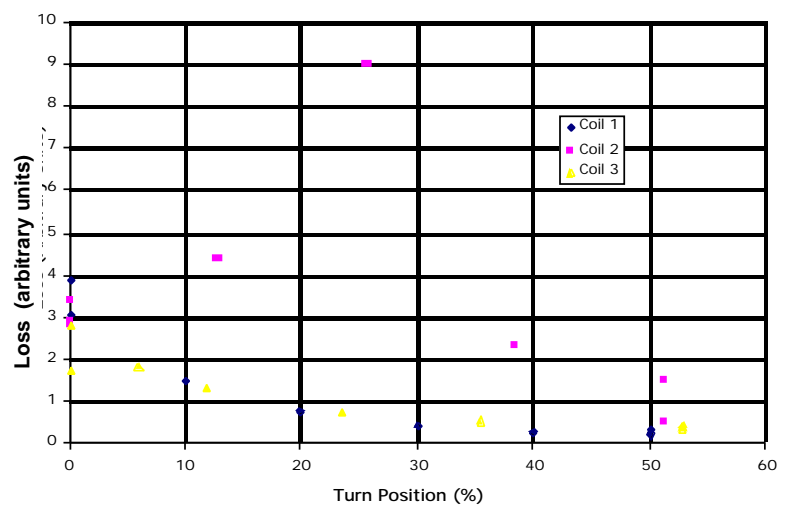

Fig. 6. Test coil losses per unit length vs. turn position.

\section{DISCUSSION}

The ac loss data for the central turns of the test coils were compared to two idealized theoretical models that give upper and lower limits for the expected losses for each winding scheme, assuming a uniform critical current in the HTSC material. The details of these calculations are proprietary. However, we can report that the measured ac losses vs. current on Test Coil 2, which used winding configuration 2, did not compare favorably with the theoretical models. The tests with configuration 1 for Test Coils 1 and 3 were close to the calculated lower limit values. While the purpose of this paper is mainly to describe experimental apparatus and experimental and analytical approach, we would note in passing that these measurements have guided and to a large degree substantiated the basic winding design and projected losses for the 5/10 MVA HTS transformer. In the future, more test coils will be investigated, and variable frequency tests will be performed to determine the relative roles of coupling and hysteresis in the ac losses. It should also be possible to perform electrical loss measurements on test coils in the same apparatus with a bridge circuit and lockin amplifier.

\section{CONCLUSION}

Calorimetric ac loss tests have been performed using a new and versatile apparatus incorporating a cryocooler. Stable temperatures between 20 and $40 \mathrm{~K}$ were achieved with unattended overnight cooldowns. Three coils were tested, allowing performance comparisons between two different BSCCO conductors and two alternative winding schemes. Use of short current pulses allowed resolution of losses on individual turns of the test coils. As expected, the observed losses were much higher on the end turns than in the center of the coils, due to perpendicular field components near the ends. Details of the loss results are confidential. Test results have been presented in arbitrary units to accomplish the main purpose of this paper, that is, to illustrate the functioning of the apparatus and the experimental and analytical approaches taken. We note in passing that these test results have been important in guiding the design of the 5-MVA transformer toward acceptable levels of ac losses.

\section{REFERENCES}

[1] S.P. Mehta, N. Aversa, and M.S. Walker, "Transforming transformers," IEEE Spectrum, vol. 34, pp. 43-49, July 1997.

[2] S.W. Schwenterly et al., "Performance of a 1-MVA HTS demonstration transformer," IEEE Trans. Appl. Supercond., vol. 9, pp. 680-684, June 1999.

[3] S.P. Mehta et al., "Progress in the design of a 5-10 MVA class HTS transformer," presented at the 2000 Applied Superconductivity Conference, Virginia Beach, VA, September, 2000.

[4] C. Schmidt and E. Specht, "AC loss measurements in the microwatt range," Rev. Sci. Instr., vol. 61, pp. 988-992, Mar. 1990. 
2000 Applied Superconductivity Conference, Paper \# 3LF02

The submitted manuscript has been authored by a contractor of the U.S. Government under contract DE-AC0500OR22725. Accordingly, the U.S. Government retains a nonexclusive, royalty-free license to publish or reproduce the published form of this contribution, or allow others to do so, for U.S. Government purposes. 